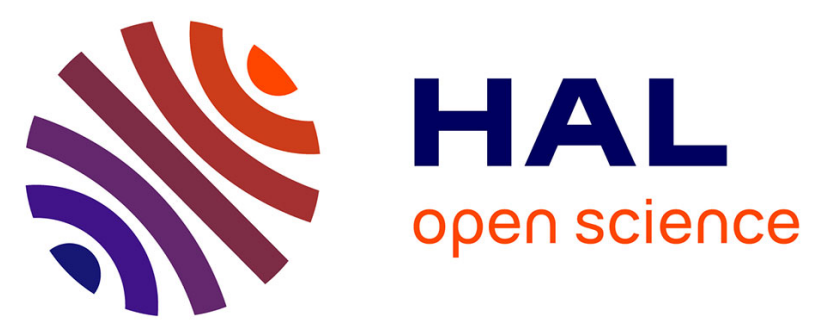

\title{
Direct Observation of Radiation-Belt Electron Acceleration from Electron-Volt Energies to Megavolts by Nonlinear Whistlers
}

F.S. Mozer, O Agapitov, V Krasnoselskikh, S Lejosne, G.D. Reeves, I Roth

\section{- To cite this version:}

F.S. Mozer, O Agapitov, V Krasnoselskikh, S Lejosne, G.D. Reeves, et al.. Direct Observation of Radiation-Belt Electron Acceleration from Electron-Volt Energies to Megavolts by Nonlinear Whistlers. Physical Review Letters, 2014, 113 (3), pp.035001. 10.1103/PhysRevLett.113.035001. insu-01256166

\section{HAL Id: insu-01256166 \\ https://hal-insu.archives-ouvertes.fr/insu-01256166}

Submitted on 14 Jan 2016

HAL is a multi-disciplinary open access archive for the deposit and dissemination of scientific research documents, whether they are published or not. The documents may come from teaching and research institutions in France or abroad, or from public or private research centers.
L'archive ouverte pluridisciplinaire HAL, est destinée au dépôt et à la diffusion de documents scientifiques de niveau recherche, publiés ou non, émanant des établissements d'enseignement et de recherche français ou étrangers, des laboratoires publics ou privés.

\section{(ㅇ)(1) $\$$}

Distributed under a Creative Commons Attribution - NonCommercial - NoDerivatives 44.0 


\title{
Direct Observation of Radiation-Belt Electron Acceleration from Electron-Volt Energies to Megavolts by Nonlinear Whistlers
}

\author{
F. S. Mozer, ${ }^{1}$ O. Agapitov, ${ }^{1,4}$ V. Krasnoselskikh, ${ }^{2}$ S. Lejosne, ${ }^{1}$ G. D. Reeves ${ }^{3}$ and I. Roth ${ }^{1}$ \\ ${ }^{1}$ Space Sciences Laboratory, University of California, Berkeley, California 94720, USA \\ ${ }^{2}$ Laboratoire de Physique et de Chimie de l'Environnement et de l'Espace (LPC2E), CNRS, Orleans 45171, France \\ ${ }^{3}$ Space and Atmospheric Sciences Group, Los Alamos National Laboratory, Los Alamos, New Mexico 87545, USA \\ ${ }^{4}$ Taras Shevchenko National University of Kyiv, Kyiv 01601, Ukraine
}

(Received 29 March 2014; published 14 July 2014)

\begin{abstract}
The mechanisms for accelerating electrons from thermal to relativistic energies in the terrestrial magnetosphere, on the sun, and in many astrophysical environments have never been verified. We present the first direct observation of two processes that, in a chain, cause this acceleration in Earth's outer radiation belt. The two processes are parallel acceleration from electron-volt to kilovolt energies by parallel electric fields in time-domain structures (TDS), after which the parallel electron velocity becomes sufficiently large for Doppler-shifted upper band whistler frequencies to be in resonance with the electron gyration frequency, even though the electron energies are kilovolts and not hundreds of kilovolts. The electrons are then accelerated by the whistler perpendicular electric field to relativistic energies in several resonant interactions. TDS are packets of electric field spikes, each spike having duration of a few hundred microseconds and containing a local parallel electric field. The TDS of interest resulted from nonlinearity of the parallel electric field component in oblique whistlers and consisted of $\sim 0.1 \mathrm{msec}$ pulses superposed on the whistler waveform with each such spike containing a net parallel potential the order of $50 \mathrm{~V}$. Local magnetic field compression from remote activity provided the free energy to drive the two processes. The expected temporal correlations between the compressed magnetic field, the nonlinear whistlers with their parallel electric field spikes, the electron flux and the electron pitch angle distributions were all observed.

DOI: 10.1103/PhysRevLett.113.035001

PACS numbers: 94.05.-a, 52.30.-q, 95.30.Qd, 96.50.Pw
\end{abstract}

Rapid acceleration of electrons up to relativistic energies occurs in different plasma configuration on all scales from the laboratory to astrophysics. The Van Allen radiation belts around Earth contain such relativistic electrons that are trapped in Earth's magnetic field. Because of intrinsic interest in the acceleration mechanism, because these electrons may be prototypical of relativistic electron acceleration in other environments, and because they present a danger to space travelers and spacecraft, it is important to understand their origin and acceleration. Two possible sources of these electrons that have been discussed are injections into the local environment of electrons that were energized by moving earthward from the tail into a stronger magnetic field while conserving their first two adiabatic invariants [1], and local acceleration in the region of the satellite measurements. While both mechanisms occur, the local acceleration mechanism has been shown to be more important, at least for major, rapid, relativistic flux increases [2-4]. Simulations of relativistic electron acceleration via the whistler mode resonance have produced relativistic electrons from seed populations of hundreds of $\mathrm{keV}$ electrons [5,6]. This work has left open the question of the source of such seed populations.

Meanwhile, observations have been made in Earth's radiation belts of parallel (to the local magnetic field) electric fields in the form of packets of spikes, each spike having a duration the order of $100 \mathrm{msec}$, and each packet containing hundreds of such spikes [7]. These spikes, dubbed timedomain structures, have at least five different forms that satisfy the above description and they have been suggested as the mechanism for producing the $\sim 100 \mathrm{keV}$ electrons that are the seed population for whistler wave acceleration to highly relativistic energies [7]. This suggestion has not been verified by detailed comparison of particles and fields before the studies described in this Letter that show, for the first time, both that low energy electrons can be accelerated up to $\mathrm{keV}$ energies by the parallel electric fields in time-domain structures and that such $\mathrm{keV}$ electrons can be further accelerated to relativistic energies via the whistler mode resonance even though their initial energies are significantly less than $\sim 100 \mathrm{keV}$. The data in this Letter were collected on May 2, 2013 on Van Allen probe B (VAP-B) by the electric field experiment [8], the magnetic field experiment [9], and the Energetic particle, Composition, and Thermal plasma (RBSP-ECT) Suite experiments [10-12]. The spacecraft was at a magnetic latitude of $2^{\circ}$, a magnetic local time of midnight, and a geocentric radial distance of 5.8 Earth radii during these measurements.

Figure 1 illustrates three components of the electric and magnetic fields in the background-magnetic-field-aligned coordinate system during a $20 \mathrm{msec}$ interval in which two packets of nonlinear whistlers were observed. Panels 1(a) and 1(b) give the two perpendicular (to the background magnetic 


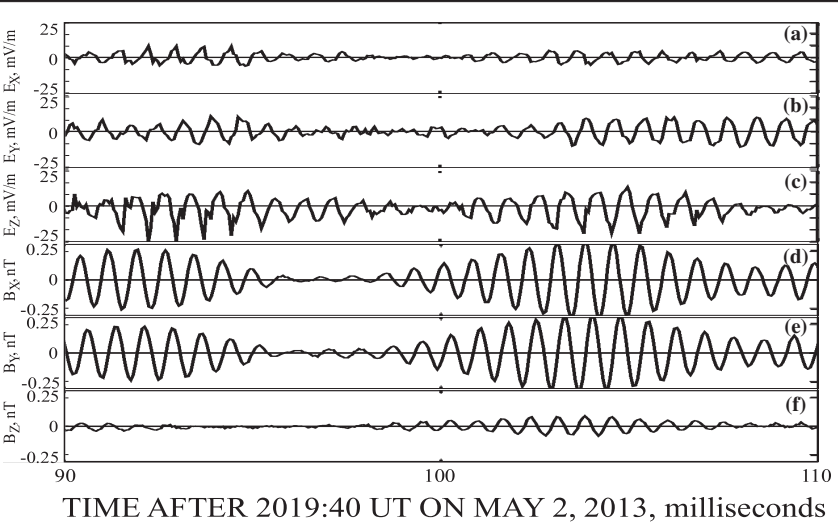

FIG. 1. Twenty milliseconds of whistler electric and magnetic field data showing nonlinear spikes in the parallel electric field that produce net parallel potentials.

field) components of the electric field, panel 1(c) gives the parallel component, and panels 1(d) and 1(e) give the two perpendicular components of the magnetic field while panel 1(f) gives the parallel component. In Fig. 1(c) there are two packets of whistler mode waves having TDS spikes that are superposed on the main whistler wave. The spikes are the negative excursions that occur once each whistler period. Because the wave period is about $0.8 \mathrm{msec}$ and the spikes have durations of a fraction of a half cycle of the wave, their durations are $\sim 0.12 \mathrm{msec}$. In the absence of these spikes, the net parallel electric field in the wave would be zero and such a wave cannot accelerate low energy electrons. If the spikes move at a speed of $50000 \mathrm{~km} / \mathrm{sec}$ (the $E / B$ ratio), each $0.12 \mathrm{msec}$ duration spike would contain a net potential of $\sim 50 \mathrm{~V}$. Data such as these were collected in short bursts covering less than a few percent of the time during the hour of interest discussed below.

The source of free energy that drives the formation and nonlinear evolution of the whistlers and TDS is the compressed magnetic field illustrated in Figs. 2(a)-2(c), which give the components of the deviations of the background magnetic field from a Tsyganenko model [13] in a magnetic-field-aligned coordinate system. During the time of interest from about 2000 to 2020 Universal Time (UT), the field increased, primarily in the nominal magnetic field direction (the $z$ direction), signifying a compression of the magnetic field that resulted from an injection event further down the tail. Injection events carry particles and electromagnetic energy earthward from the nightside tail during magnetic activity. Evidence that the electrons from this injection event did not reach VAP-B and that the electron flux increases described below came from local acceleration is given in Fig. 3, in which the phase space density of the average of the 54 and $75 \mathrm{keV}$ electrons observed on VAP-B at a radial distance of 5.8 Earth radii was much greater than that of the measured of 51-72 keV electrons on Los Alamos satellite LANL-04A, located about 0.8 Earth radii tailward of VAP-B and within $1 \mathrm{~h}$ of local time. (Because of the finite energy steps of the measurements it is not possible to obtain the two phase space densities at the same values of the first invariant, so average values of the VAP data are used.) By Liouville's theorem, the VAP-B electrons must have been accelerated earthward of LANL04A and could not have come from the magnetospheric tail.

An important feature of the magnetic field in Figs. 2(a)-2(c) is the bipolar (plus and minus) deviation

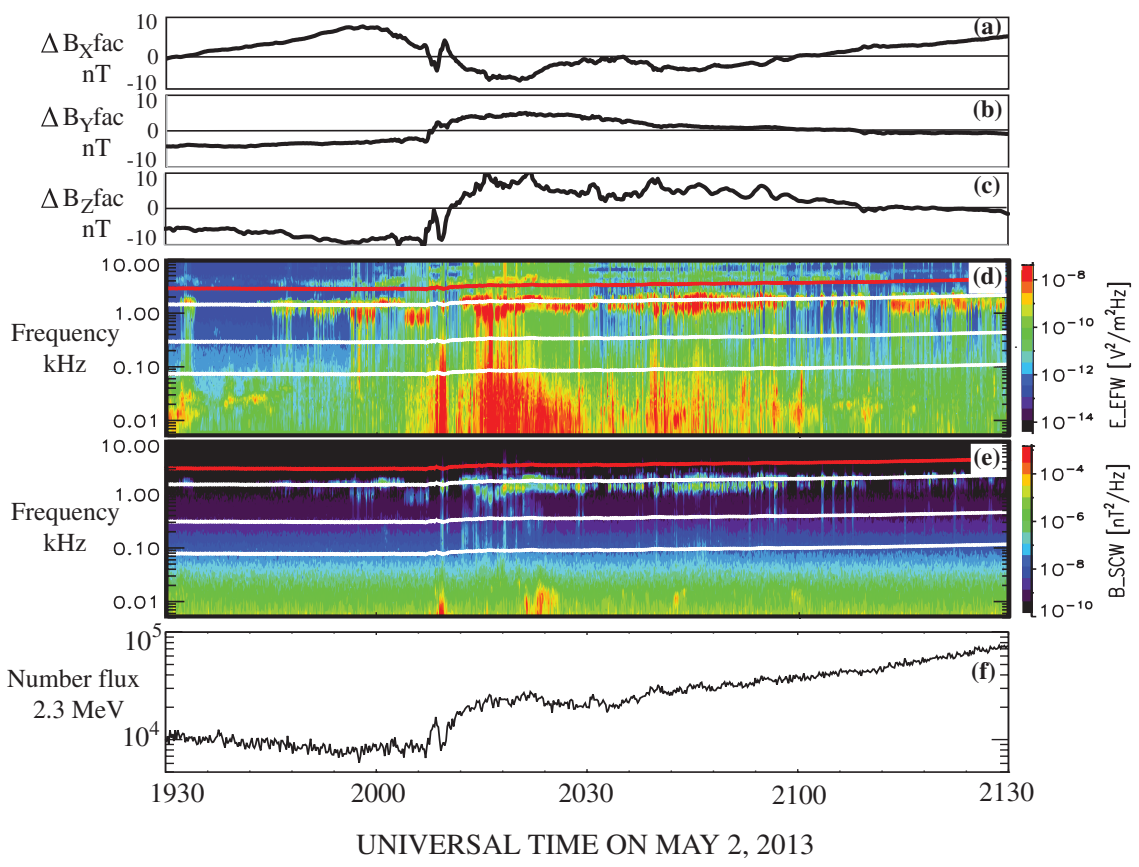

FIG. 2 (color). (a)-(c) Components of $\Delta B$ in magnetic field aligned coordinates. (d)-(e) Spectra of the total electric and magnetic fields, respectively. (f) The number flux of $2.3 \mathrm{MeV}$ electrons, which shows that the acceleration processes being discussed also produced relativistic electrons. 


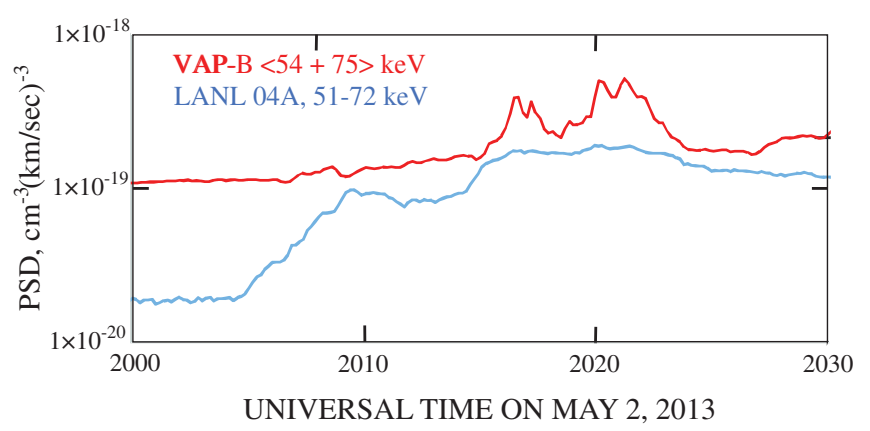

FIG. 3 (color). Phase space densities as a function of time for electrons observed on VAP-B and LANL-04A, where VAP-B was about 0.8 Earth radii earthward of LANL-04A.

between about 2004 and 2012 UT, which is the signature of a field-aligned current. Such currents can cause important wave activity when the relative velocity of the current-carrying electrons exceeds a threshold associated with current driven instabilities. This current may provide the free energy that results in the TDS and intense whistler waves that are illustrated in Figs. 2(d) (electric field spectra) and 2(e) (magnetic field spectra). Whistlers were observed (at the top white line in Fig. 2(d), which is half of the electron gyrofrequency) during the central hour of this interval. Also, broadband electrostatic noise (below $1 \mathrm{kHz}$ ) was observed in electric field panel 2(d) during this time. This noise was first observed in 1974 [14] and identified as the signature of stochastically occurring TDS in 1994 [15]. We have also shown the correlation of this noise with TDS in the present data from bursts occurring between 2014 and 2018 UT and, again, between 2039 and 2048 UT, as well as in dozens of other Van Allen probe examples. From this broadband electrostatic noise it is concluded that nonlinear whistlers, like those in Fig. 1, existed for a significant fraction of the time after 2000 UT. The nonlinear whistlers accelerated electrons to relativistic energies, as is discussed below and shown in panel 2(f), in which the flux of $2.3 \mathrm{MeV}$ electrons increased by almost an order of magnitude during the time of interest.

The whistler perpendicular electric field amplitude is given in Fig. 4(a). (The absolute amplitude obtained from the time-domain bursts is given by the red crosses and a broadband filter output, which is an underestimate of the signal for a pure sine wave, is given as the solid black data.) The broadband electrostatic noise amplitude at $300 \mathrm{~Hz}$, due to the nonlinear TDS, is given in Fig. 4(b). Figure 4(c) gives plots of the electron number flux (counts $/ \mathrm{cm}^{2} \mathrm{sec}$ ster $\mathrm{keV}$ ) at all energies measured by the HOPE and MAGEIS plasma instruments. At precisely the onset of the TDS after 1950 UT [Fig. 4(b)], the flux of the lowest energy electrons decreased [top six plots in Fig. 4(c)] because these electrons were accelerated to higher energies, while the flux at higher energies increased. The increased flux of electrons with energies below a few $\mathrm{keV}$ and due to the TDS was largely completed by about 2008 UT, after which they were depleted by the higher energy acceleration in the presence of increased amplitude whistler waves [Fig. 4(a)]. The relatively rapid flux increase of the few $\mathrm{keV}$ electrons was due to the fact that they were the first particles to experience the whistler resonance interaction. Overall, the flux increases occurred in the sequence of lower energy

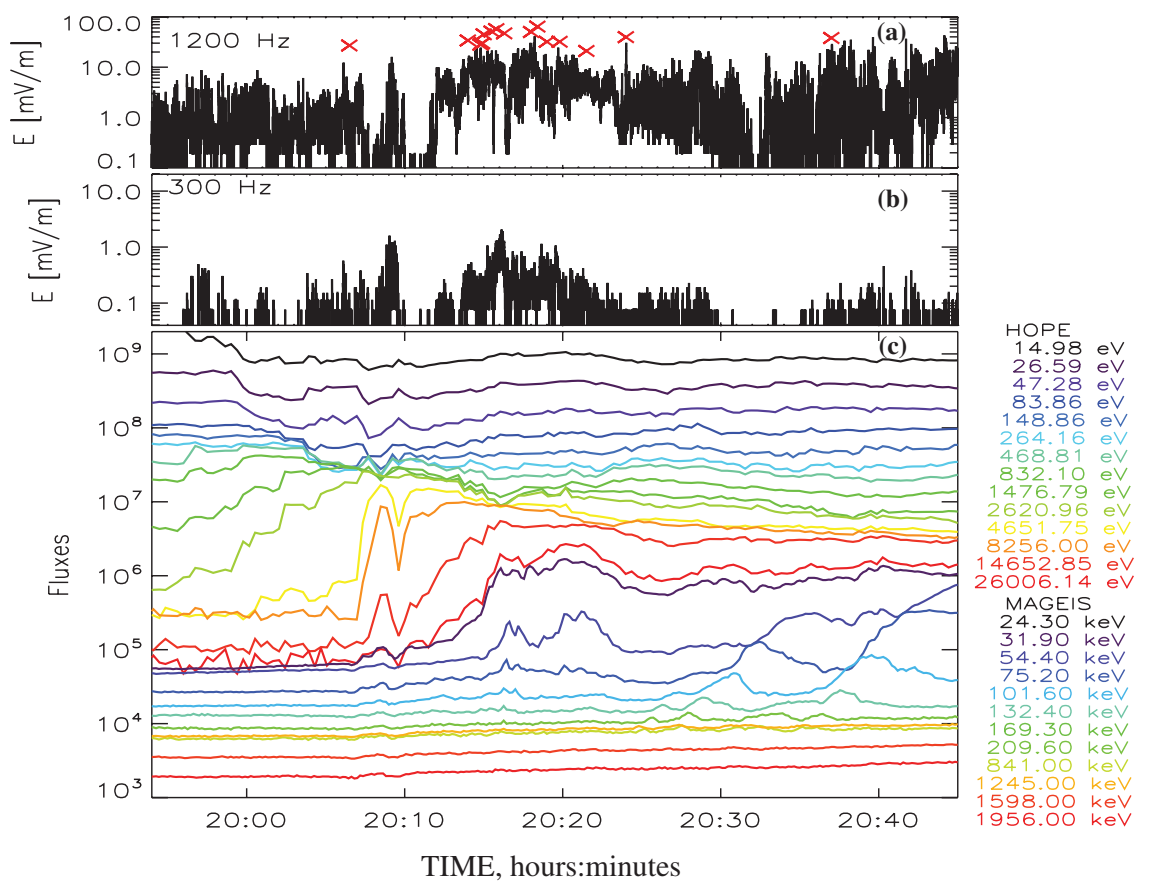

FIG. 4 (color). (a) The whistler electric field amplitude as a function of time. (b) The electric field power at $300 \mathrm{~Hz}$, which is a proxy for the occurrence frequency of the TDS. (c) The electron number flux (counts $/ \mathrm{cm}^{2}$ ster sec keV) measured by the particle experiments on the satellite. Because the spacecraft was at a positive potential of 6-8 V during these measurements, the actual electron energies are $6-8 \mathrm{eV}$ greater than given in the figure. 
increases before higher energy increases. This suggests that the observations resulted from local acceleration and not from electrons accelerated at another local time and that drifted to the spacecraft location because, in the latter case of longitudinal drift, the higher energy electrons would be observed before those of lower energy.

The conclusion of local acceleration of low energy electrons to $\mathrm{keV}$ energies in the net parallel electric field of the nonlinear whistlers is supported by the pitch angle distributions of these and more energetic electrons illustrated in Fig. 5. The 0.105, 0.264, and $0.661 \mathrm{keV}$ electrons (top three panels of Fig. 5) were accelerated by the parallel electric field in the nonlinear whistlers to produce a decrease of perpendicular fluxes and an increase of parallel fluxes with time. That the low energy electrons became field aligned at the same time that the TDS waveforms appeared in Fig. 4(b) makes explanations of the field-aligned pitch angle distributions by any other imagined mechanism than acceleration by the parallel electric fields in the spikes of Fig. 1 highly unlikely. By contrast to the low energy parallel acceleration, the increases of the higher energy electron fluxes (bottom two panels of Fig. 5) were at pitch angles closer to $90^{\circ}$. This result shows two different acceleration processes at work. The first mechanism involved acceleration to $\mathrm{keV}$ energies by parallel electric fields in TDS that produced field-aligned pitch angle distributions (top three panels of Fig. 5). The second mechanism involved acceleration from $\mathrm{keV}$ energies to $\mathrm{MeV}$ energies by means of particle energy growth or diffusion due to the cyclotron resonance [Fig. 2(f) and the bottom two panels of Fig. 5]. An electron, traveling along the magnetic field in the opposite direction of a propagating whistler, has the same sense of gyration about the magnetic field line as does the whistler, so it can be accelerated by the perpendicular electric field in the whistler if the whistler's Doppler shifted frequency is the same as the electron gyration frequency.

A surprising observation in the current data is that perpendicular acceleration by the cyclotron resonance was observed at energies as low as $2.6 \mathrm{keV}$. It is usually supposed that this process operates only for much higher initial energies [5,6]. However, in this case, the waves had characteristic frequencies in the upper band of the chorus spectrum with typical wave frequencies of 0.7 times the electron gyrofrequency [see Fig. 2(d)], and this allowed lower energy particles to be resonant with the whistlers [16]. To illustrate why this happens, consider the condition for an electron's gyrofrequency to be the same as the whistler's Doppler shifted frequency,

$$
\mathrm{v}_{\|}=(\Omega / \gamma-\omega) / \mathrm{k}_{\|},
$$

where $\mathrm{v}_{\|}$is the parallel electron speed, $\mathrm{k}_{\|}$is the parallel wave number, $\Omega$ is the electron cyclotron frequency $(e B / m c$, where $\mathbf{B}$ is the magnetic field and $m$ is the electron mass), $\gamma$ is the relativistic factor, and $\omega$ is the whistler wave frequency. That such low energy electrons can participate in the cyclotron resonant interaction follows from the fact that the right side of the above equation (and, hence, the required parallel electron velocity) gets smaller as the whistler frequency approaches the cyclotron frequency. In addition, the cold plasma whistler dispersion relation for parallel propagating waves, $k^{2} c^{2}=\omega^{2}+\omega_{p}^{2} \omega /(\Omega-\omega)$, (where $\omega_{p}$ is the electron plasma frequency, equal to $\left(4 \pi n e^{2} / \mathrm{m}\right)^{1 / 2}$, and $n$ is the electron density) requires that $k_{\|}$become larger as $\omega$ approaches $\Omega$, so the required $v_{\|}$in Eq. (1) becomes even smaller. Thus, the solution to Eq. (1) is that a one $\mathrm{keV}$ parallel propagating electron can satisfy the cyclotron resonance condition at an equatorial geocentric distance of six Earth radii for a whistler frequency greater than $0.62 \Omega$. The acceleration of low energy electrons in the current data stems from the fact that $\omega / \Omega$ was as large as 0.7 .

That such low energy electrons can be accelerated to relativistic energies by a small number of resonant interactions with the whistler is shown by the test particle simulation described in Fig. 6, which is similar to previous

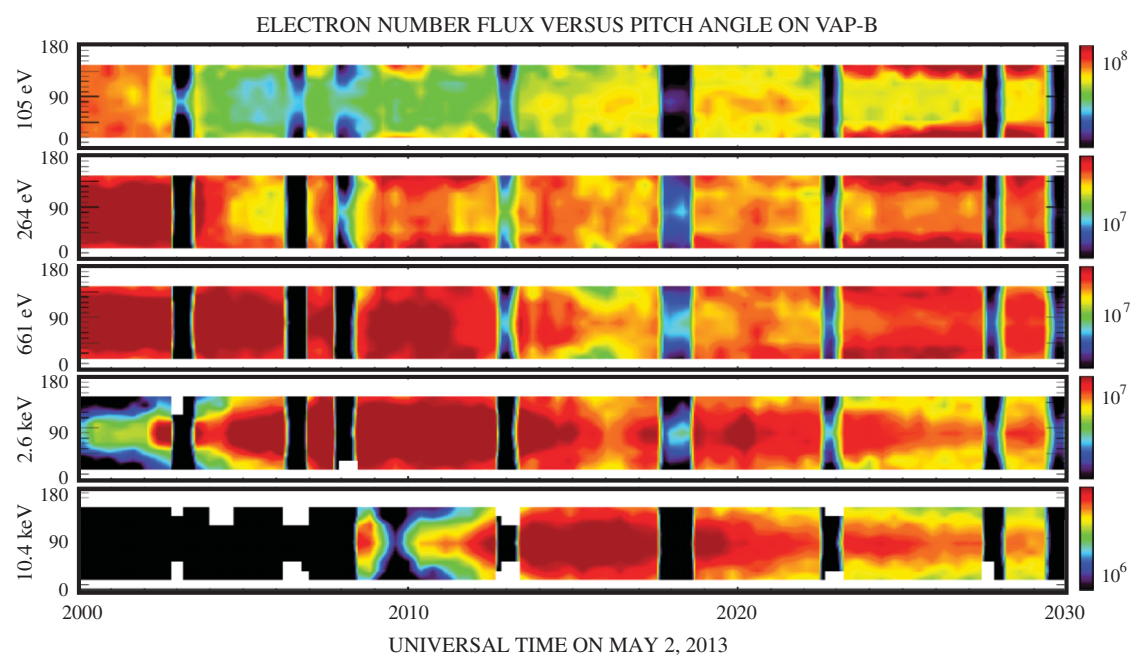

FIG. 5 (color). Pitch angle distributions as a function of time for electrons with energies from 0.1 to $10.4 \mathrm{keV}$. The dynamic range of the color scale in all plots is a factor of 10. 


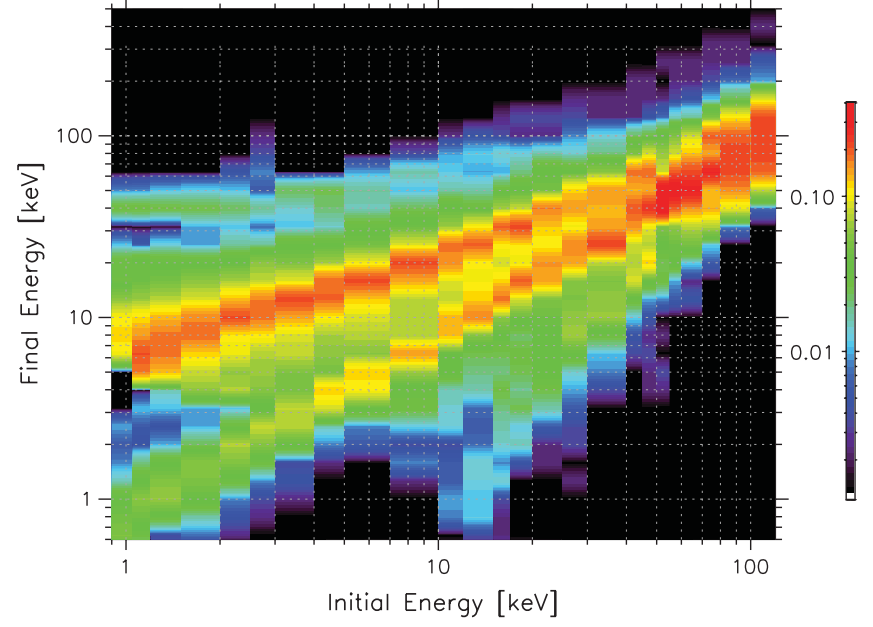

FIG. 6 (color). Test particle calculations showing the range of output energies achieved by incident electrons with fixed energies and random phases with respect to the whistler wave with which they are in resonance.

work [17-19] and that shows the energy range of electrons that have undergone several acts of trapping-acceleration detrapping in a parallel propagating sine wave having a $100 \mathrm{mV} / \mathrm{m}$ parallel electric field and a $0.4 \mathrm{nT}$ magnetic field. The energy acquired by a resonant electron of a fixed energy in a single interaction with the whistler depends on its gyrophase with respect to that of the whistler. Test particle calculations of the acceleration of variable phase, fixed energy, incident electrons in a $100 \mathrm{mV} / \mathrm{m}$ wave having $\omega / \Omega=0.7$, in a $140 \mathrm{nT}$ equatorial, dipole magnetic field are shown in Fig. 6. Final energies of electrons having an initial energy given by the abscissa of Fig. 6 are presented in the ordinate of Fig. 6. The colors in this figure delineate the relative number of electrons at each output energy for the given input energy. For example, an initial $1 \mathrm{keV}$ electron has a likely energy after a single resonant interaction of $10 \mathrm{keV}$ and a maximum energy of $70 \mathrm{keV}$. Similarly, a $10 \mathrm{keV}$ incident electron has a likely output energy of $25 \mathrm{keV}$ and a maximum energy of $100 \mathrm{keV}$, while an incident $100 \mathrm{keV}$ electron has a likely output energy of $150 \mathrm{keV}$ and a maximum energy of $400 \mathrm{keV}$. Thus, this simulation shows that electrons which were accelerated to $\sim 1 \mathrm{keV}$ in the parallel electric field of TDS, can be further accelerated to relativistic energies in a small number of cyclotron resonant interactions with the whistler wave, just as observed experimentally in Figs. 4 and 5. That such an electron can undergo several interactions as it gains energy is shown by the range of energies along the abscissa of Fig. 6 that are in resonance with the whistler.

Large-amplitude whistler waves observed in this region can be associated with local compression of the magnetic field, as discussed previously [20]. After the local compression in the magnetic field of Figs. 2(a)-2(c) at 2010 UT, amplification of the chorus amplitude by a factor $\sim 3$ was observed in Fig. 4(a). The two possible mechanisms that can be responsible for this amplification are temperature anisotropy and other features of the electron distribution function [18]. When the wave amplitude grows to large values, an important part of the plasma distribution begins to be trapped and the waves evolve and form nonlinear wave packets with nonzero parallel components. Thus, the same whistler waves that resonate with the electrons can play an important role in the evolution of TDS, accelerating their steepening and amplifying their electric field amplitude through wave-wave interactions.

The authors thank the very large numbers of people who built the scientific instruments and the Van Allen probes as well as the spacecraft operators and programmers who developed the data analysis software. We acknowledge LANL for provision of measurements on board geostationary satellites. This work was performed under JHU/APL Contract No. 922613 (RBSP-EFW). V. K. is grateful to CNES for financial support through the Grant "Modele d'ondes."

[1] M. Shulz and L. J. Lanzerotti (Springer, New York, 1975).

[2] R. S. Selesnick and J. B. Blake, J. Geophys. Res., 105, 2607 (2000).

[3] Y. Chen, G. D. Reeves, and R. H. W. Friedel, Nat. Phys. 3, 614 (2007).

[4] G. D. Reeves et al., Science 341, 991 (2013).

[5] I. Roth, M. Temerin, and M. K. Hudson, Ann. Geophys., 17, 631 (1999).

[6] Y. Omura, N. Furuya, and D. Summers, J. Geophys. Res., 112 A06236 (2007).

[7] F. S. Mozer, S. D. Bale, J. W. Bonnell, C. C. Chaston, I. Roth, and J. Wygant, Phys. Rev. Lett. 111, 235002 (2013).

[8] J. R. Wygant, J. W. Bonnell, K. Goetz, R. E. Ergun, F. S. Mozer, S. D. Bale, M. Ludlam, P. Turin, P. R. Harvey, and R. Hochmann, Space Sci. Rev. 179, 183 (2013).

[9] C. A. Kletzing, W. S. Kurth, M. Acuna, R. J. MacDowall, R. B. Torbert, T. Averkamp, D. Bodet, S. R. Bounds, M. Chutter, and J. Connerney, Space Sci. Rev. 179, 127 (2013).

[10] H. O. Funsten, R. M. Skoug, A. A. Guthrie, E. A. MacDonald, J. R. Baldonado, R. W. Harper, K. C. Henderson, K. H. Kihara, J. E. Lake, and B. A. Larsen, Space Sci. Rev. 179, 423 (2013).

[11] J. B. Blake et al., Space Sci. Rev. 179, 383 (2013).

[12] D. N. Baker, S. G. Kanekal, V.C. Hoxie, S. Batiste, M. Bolton, X. Li, S. R. Elkington, S. Monk, R. Reukauf, and S. Steg, Space Sci. Rev. 179, 337 (2013).

[13] N. A. Tsyganenko, H. J. Singer, and J. C. Kasper, J. Geophys. Res. 108, 1209 (2003).

[14] F. Scarf et al., J. Geophys. Res. 1, 189 (1974).

[15] H. Matsumoto, H. Kojima, T. Miyatake, Y. Omura, M. Okada, I. Nagano, and M. Tsutui, Geophys. Res. Lett. 21, 2915 (1994).

[16] B. Ni, R. M. Thorne, Y. Shprits, and J. Bortnik, Geophys. Res. Lett. 35, L11106 (2008).

[17] T. F. Bell, J. Geophys. Res. 89, 905 (1984).

[18] V. Y. Trakhtengerts, M. J. Rycroft, D. Nunn, and A. G. Demekhov, J. Geophys. Res. 108, 1138 (2003).

[19] J. Bortnik, R. M. Thorne, and U. S. Inan, Geophys. Res. Lett. 35, L21102 (2008).

[20] W. Li et al., J. Geophys. Res. 114, A00C14 (2009). 